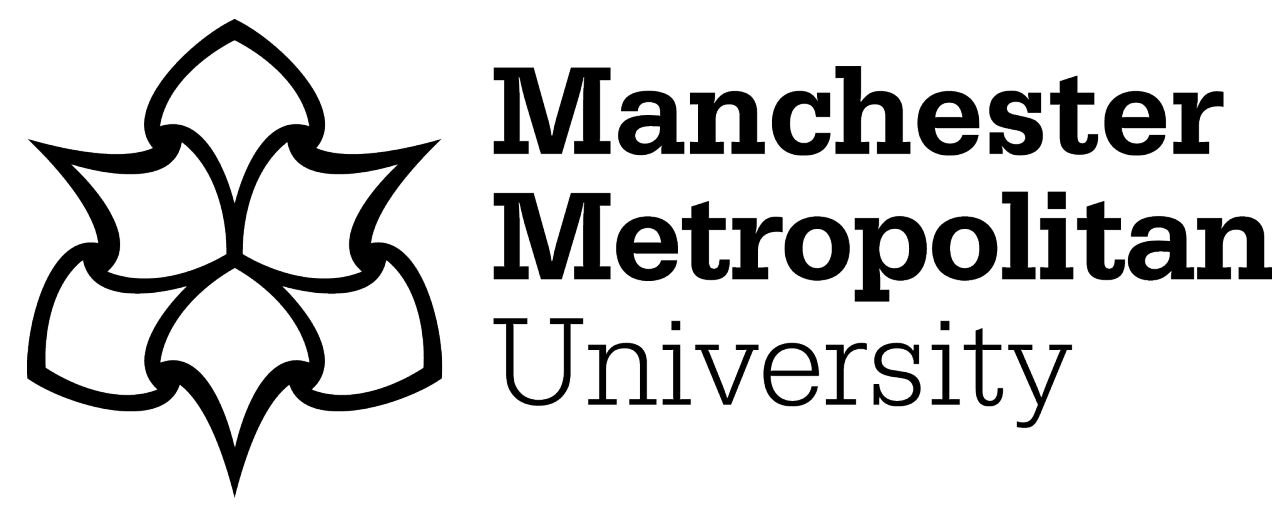

Ward, R and Campbell, S ORCID logoORCID: https://orcid.org/0000-00022920-7318 (2013) Mixing methods to explore appearance in dementia care. Dementia, 12 (3). pp. 337-347. ISSN 1471-3012

Downloaded from: https://e-space.mmu.ac.uk/625040/

Version: Accepted Version

Publisher: Sage

DOI: https://doi.org/10.1177/1471301213477412

Please cite the published version 


\title{
Mixing methods to explore appearance in dementia care
}

\author{
Richard Ward \\ University of Stirling, UK
}

\section{Sarah Campbell}

University of Manchester, UK

\begin{abstract}
This paper considers approaches to investigating appearance and the work invested in maintaining it within dementia care. Our focus is upon methodological and methods-related issues associated with the challenge of generating knowledge of the embodied worlds of people with dementia. We begin with a brief overview of the literature on appearance and dementia, and consider what it teaches us about the nature of appearance and ways of understanding it. We describe and discuss the mixing of methods for an on-going investigation into hairdressing in dementia care: The Hair and Care project. Based upon the experience of research in care-based hair salons, we argue for a creative use of methods in dementia studies as an avenue to better engaging with the embodied experiences of people with dementia and, as a result, understanding how people use their bodies and senses to create meaningful worlds.
\end{abstract}

\section{Keywords}

appearance, body work, dementia, embodiment, qualitative methods

\section{Introduction}

Across the social sciences, a commitment to taking account of the body in social theory and research is now well established (e.g. Butler, 1993; Grosz, 1994; Shilling, 2003; Turner, 1984). Image and appearance have been an important feature of the ensuing debate, not least in explorations of the ageing body and the politics of age (e.g. Biggs, 1997; Bytheway \& Johnson, 1998; Coupland, 2003; Faircloth, 2003; Featherstone and Hepworth, 1991; Furman, 1997; Twigg, 2007; Ylanne, 2012). Yet, in dementia studies, appearance has received limited attention and where it has, is often treated as an aside to discussions of care practice rather than being a focus in its own right. Ways of understanding the body in studies on dementia seem to have ruled out interest in appearance and underplayed the significance to people's lives in dementia care. 
In this paper, we introduce an on-going investigation into appearance and the work invested in it, in dementia care. As the name suggests, the Hair and Care project has focused particularly upon hair and how it is styled and managed and the meanings this holds for workers and people with dementia. Here, we discuss a mixed method approach to researching appearance, developed for the project. We begin with a short review of the still limited literature on appearance and dementia and highlight certain key messages from this body of work. On the basis of this reading, we set out the understanding of appearance that we have used to organise our own research and the thinking behind this. We then spotlight three of the main methods we have used to generate knowledge about hairdressing and appearance in dementia care before considering what this particular mix of methods adds to an existing understanding of appearance in a dementia care context.

\section{Understanding appearance in the context of dementia care}

While investigations into appearance remain limited in dementia studies, existing research reveals that it is integral to the everyday experiences of people with dementia. There are three distinctive themes within this work. Firstly, research has considered questions over the authorship of appearance and the often uneasy bodily negotiations that occur when care staff assume responsibility for certain appearance-related practices, often under the watchful gaze of families and friends. Secondly, attention has been directed to the efforts of people with dementia to maintain their own appearance and consideration given to what this means for selfhood and identity. Thirdly, research has begun to explore the role of appearance in the relationship between people with dementia and the material environment that they inhabit and the opportunities that arise from this.

Efforts to explore bodies as the object of caring practices have revealed how the more malleable aspects of appearance - hairstyle, dress/dressing and make-up - are often subsumed into formal caring routines in ways that inscribe the body with meanings reflective of institutional priorities. By spotlighting the figure of the 'lounge-standard resident', Lee-Treweek $(1994,1997)$ has shown how bodies are territorialised through care work as the efforts of staff centre upon the maintenance of 'some semblance of gendered normality through dressing, applying make-up, combing hair and putting in dentures' (1997, p. 53). Lee-Treweek's analysis underlines the embodied vulnerabilities of people in care. She demonstrates that individual appearances can be usurped for institutional purposes to signify the completion and quality of care but at the risk of erasing the identities of care recipients. Ward, Vass, Aggarwal, Garfield and Cybyk (2008) similarly found that bodies are contested territory in dementia care settings. The appearance of the cared-for is invested with meanings that reflect the differing perspectives of workers and families. Often, appearance became a focus for tensions between the practicalities of care provision and a desire for biographical continuity in how a person looked. Such analyses point not only to the symbolic significance of appearance in dementia care but also the need to consider it as a locus for social and political control (Katz, 1996). Crucially, this work opens up questions concerning the aim or purpose of the support given to people with dementia to maintain their appearance. A certain standard of smartness and presentation is generally applied to people in care - but should this be the goal of appearance-related body work?

Existing research has also considered the efforts of people with dementia to maintain their appearance. In developing a theory of 'embodied selfhood', Kontos $(2004,2005,2006)$ draws attention to everyday acts of appearance-maintenance as on-going expressions of self. 
Combining attention to the 'existential immediacy of the body' (2006, p. 196) with recognition of a person's own embodied history, Kontos argues that selfhood resides in everyday human gestures and movements - such as the act of pausing to apply lipstick before entering a room. Her analysis underlines the agentive and self-sustaining qualities of appearance-related practices. Appearance is understood as culturally and biographically expressive; gendered performances rooted in long-standing processes of socialisation and enculturation.

In her work on clothing and dementia, Twigg (2010, see also Twigg and Buse in this edition) shows how appearance extends to include the body's relationship with the objects and possessions which we order and organise as expressions of our identities.

How we sit or move is affected by the clothes we wear: for women, trousers prompt different ways of sitting and walking from a skirt; you dispose of your limbs in a long loose dress differently from a short tight one; a shawl endorses a particular range of gestures. What we wear is thus more than just how we present ourselves in terms of outward appearance, but part of how we enact our being. (2010, p. 225)

Twigg (2010, p. 226) argues that clothes form part of a continuing narrative of the self and suggests we understand clothing as the 'environment closest in'. Our social presence is shaped by our engagement with these possessions and this relationship provides a meaningful vehicle for the performance of identities.

In their work on clothing and dementia Iltanen-Tahkavouri, Wikberg, and Topo (2012) (see also Topo \& Iltanen-Tahkavouri, 2010) consider garments specifically designed for wear in institutionalised settings. Their work returns to questions over the authorship of appearance and the underlying ethical implications when clothing becomes a resource for care. Their research highlights that specially designed clothing can facilitate the independence of the wearer, for example in supporting the management of incontinence. But, clothing design can also delimit the agency of the wearer, for instance by positioning fasteners to prevent someone from removing their clothes. Hence, design becomes an agent in the regulation of bodies in care.

\section{Developing a focus on appearance as process in the context of dementia care}

This existing body of work provides a useful framework from which to develop a working understanding of appearance in the context of dementia care, while helping us to appreciate what is still missing from this debate. There are a series of clear messages that have informed our own research.

Firstly, through the lens of embodied selfhood, Kontos $(2004,2006)$ has made clear that everyday acts of appearance maintenance are in themselves often deeply meaningful. They constitute an expression of self and serve to uphold cultural belonging and social difference. This perspective also offers a useful basis on which to consider the implications when such appearance-related practices are assumed by care providers. The way in which this is done and the imperatives that guide this work become vital considerations in the context of this understanding of appearance. If a person's appearance becomes simply a by-product of the care they receive to what extent can we understand such care to be person-centred? Hence, understanding the negotiation of authorship behind appearances in care emerges as a crucial consideration for personhood. 
Work on clothing has foregrounded the body-practice-place relationship that underpins appearance and the meanings that emerge from it. In her analysis, Twigg (2010) draws attention not only to the embodied relationship we have to clothes, such as how different fabrics feel against the skin, but also the often personalised meanings clothes can hold, evoking memories or as gifts from loved ones. Appearance is shown to involve the interplay of the embodied and the symbolic, which challenges more narrowly 'textual' readings of how a person looks. By tracing how bodies move according to how they are clothed our attention is drawn to the performative nature of appearance. From this perspective appearance is shown to be animated and processual, dispelling notions of it is as something that is fixed, or as an endpoint to care.

However, as an emerging field of interest it is clear certain gaps exist in our understanding of appearance and dementia. Notably, despite efforts to foreground the embodied perspectives of people with dementia, their voices remain muted within the literature to date. We have yet to hear directly from people with dementia concerning their diverse experiences of appearance or the meanings they attach to it. Furthermore, little consideration has been given to how appearance feels. In particular, the sensory and affective dimensions associated with the maintenance of appearance have been overlooked with little thought given to how meaning may reside in this sensorial dimension.

The key findings from existing research and an awareness of the current gaps in our understanding have helped to inform our research and in particular have pointed to the value of a mixed methods approach designed to capture the multi-faceted nature of appearance. For the purposes of our investigation, it was clear that we needed to define appearance in a way that would incorporate these different facets and the relationships between them. Hence, rather than disentangling the way we look (image) from the work invested in maintaining it, we viewed the various practices involved in maintaining appearance as integral to our overall understanding. Indeed, in light of the emphasis on the performative and processual nature of appearance in more recent thinking, a division between image and its maintenance seemed artificial as well as unhelpful.

In the next section, we outline our research and discuss efforts to explore the multi-faceted nature of the appearance process through a mixing of qualitative methods.

\section{Introducing the Hair and Care project}

The Hair and Care project is a 28-month ESRC-funded (Economic and Social Research Council) investigation into the provision of hairdressing services to people with dementia. Hence, a particular focus for our work was the care-based hair salon. Distinct from high street salons, these spaces in care have largely escaped the attention of research in dementia studies so a central aim has been to generate a rich description of the salon process supported by an exploration of the meanings attached to appearance and the work of maintaining it by workers and people with dementia.

The research has been undertaken in a designated region of north-west England, an area which contains some of the most deprived neighbourhoods in the UK as well as more affluent areas. As we discuss below a mix of methods was used in order to explore the differing dimensions of the appearance process. This has included mapping of the provision of care-based hairdressing across the area of the study, as well as sensitising discussion groups and more in-depth interviews with stakeholders and 'key informants'. At the heart of the study has been an 8-month period of participant observation in a 
series of care-based salons in hospitals, day centres and care homes as well as visits to people at home. During these periods of observation we conducted filmed conversations with workers and their clients. In total there have been 8 observation sites and we have made repeated visits following individuals as they participate in the salon experience. A total of 47 hours of filmed data has been generated during these visits.

In the final stage of fieldwork we recruited people with dementia to participate in what we have labelled 'appearance biographies'; a life review method developed for the project (discussed further below) that focuses on appearance and the work invested in it over the life course. From the outset, it was our intention to involve people with dementia in the project irrespective of the severity of their condition. As this meant conducting research with people who may not have capacity to consent, we were required to obtain ethical approval from an approved NHS Research Ethics Committee with knowledge of the Mental Capacity Act (2005), a process we have described elsewhere (Ward and Campbell, in press).

\section{Mixing methods to deepen our understanding of the appearance process}

The research involved a mix of methods, some of which were more experimental and less familiar to the field of dementia studies. In this section we outline three of the methods used and consider their contribution to our understanding of appearance in dementia care.

In-situ conversations in the salon: A particular focus for the study was the care-based salon and the activities that took place within it. Not only is a visit to the hairdresser an integral feature of appearance maintenance for many people throughout their lives but the salon is a space in which people discuss and reflect on the way they look. We used a mix of methods to generate knowledge of the salon that included semi-structured interviews with care-based hairdressers, participant observation and filming. Initially, we used the camera to make process films of each salon, capturing the different stages of the hairdressing process, the embodied practices involved and the associated interactions. We then progressed to filming in-situ conversations with the hairdressers and their clients.

The in-situ conversations were unstructured discussions where we asked people to describe what they were doing, as they did it and to tell us how it felt. With the hairdressers this involved them in narrating their work and describing the thinking behind it. With people with dementia the encounters were more diverse. In some cases it was possible to engage people in discussions of the different sensations and physical experiences as they progressed through the hairdressing process and what these meant to them. For others, a spoken narrative was not possible, but the filming was able to capture their facial expressions, gestures, responses to sensory experiences and what Pink (2005), discussing her own use of filmed in-situ interviewing, describes as the 'embodied-enacted' (2005, p. 277) aspects of the salon. In other words, how people engaged with the material environment and used the space of the salon.

There were a number of benefits to this method in terms of deepening our understanding of appearance and the work invested in it. Firstly, we generated certain distinctive insights into hairdressing as a particular type of appearance-related body work in dementia care. In her efforts to raise the profile of body work Wolkowitz (2006) has argued for the need to listen to accounts from workers to learn about body work 'from the inside' and has highlighted the absence of evidence of what body work feels like. We found that in-situ conversations 
provided useful narratives of the process of hairdressing, while the filming captured the intercorporeal aspects of the work that were rarely touched upon during interview.

In-situ conversations had particular benefits to supporting people with dementia to participate in the research (Bamford \& Bruce 2000). This approach dispensed with the need for recall and helped to foreground the multi-sensory experience of the salon as people described such sensations as the massaging of their scalp when the shampoo was applied or the brushing of their hair at the end of the process. By filming these encounters we were able to treat them as 'ethnographic events' (Mason \& Davies 2011) in which we not only captured what people had to say but also a much wider and richer range of interactions, movements, visual and sensory dimensions. This was valuable because so much of 'the action' of the salon is unspoken, sensory and embodied but it also meant we had more clues to understanding the experiences of people who were unable to voice their perspective.

Overall, the filmed in-situ conversations underlined the processual and performative nature of appearance, they offered clues to the embodied and sensory aspects to maintaining appearance and revealed the detail of how an image or style is negotiated between worker and client.

Participating in the salon process: Another of the more experimental approaches to generating knowledge of the salon involved Sarah, the researcher on the project, asking one of the hairdressers to take her through the salon process as a client. Her hair was washed, cut, put in rollers and sprayed with setting lotion. She was then sat under the hooded hairdryer and in the final stage of the process the rollers were removed and her hair was combed out. Sarah set up the camera to record the process and discussed with the hairdresser the different stages and the associated sensations as they occurred. Here, she describes in her field-notes the experience of having her hair washed as part of this process:

The hairdresser asked me if I was ok to face forward. I realised that in salons today they are usually back washes, so this was different for me. She put a towel around me and I leant over the sink. There was lots of water rushing; it took a few moments for the temperature to become warm enough. The water rushed over me and I felt very wet, it was also very dark as I faced into the sink and couldn't open my eyes. I held the towel around my face. The shampoo massage was lovely, but I was very aware of all the water. The water was warm now, and the smells of the shampoo and conditioner filled my nose. As the hairdresser firmly lifted me up from the sink water dripped down my face. The light changed as I came up from the dark depths of the sink (12.30 pm - 24th February 2012)

Use of the body for research in this way is an established practice in anthropology and owes much to Bourdieu's (1977) theory of practice where he recommends bodily engagement rather than observation alone. Jackson (1983) has also recommended 'using one's body in the same way as others in the same environment' (1983, p. 340) as a distinctive interpretive activity. In dementia studies, McColgan (2005) (citing Okely 1978) advocates using our bodies to synchronise posture and movement with those around us to learn through our senses.

So, what were the benefits of this approach to our understanding of appearance? Firstly, it offered insights into the inter-corporeal relationship between worker and client that lies at the heart of hairdressing. We discovered how touch is used in different ways, sometimes gently to guide the body of the client and at other points firmly in order to position them and hold their bodies in place. Participation in the salon process brought to life the textures, scents, temperatures and other sensations. The rushing of the water, the gentle snip-snip-snip 
of the scissors and the hum of the hairdryer coalesced to create the hairdressing experience. This aspect of the research underlined that appearance is meaningful beyond its culturally encoded and symbolic outward markers. How appearance is felt and sensed carries meaning as well, not least in how a particular configuration of sensations, such as are involved in a visit to the salon, become sedimented in the course of a person's life as the basis for embodied memory and knowledge.

Appearance biographies. In the final phase of the fieldwork we asked people with dementia to take part in interviews to explore their 'appearance biographies', where we set out to examine appearance over the course of their lives. This was a method developed specifically for the study but which drew upon reminiscence and life story approaches. Often these interviews involved both the person with dementia and their primary carer and in many instances people chose to use family photo albums as a resource for our discussions. Not everyone referred to photographs but for some these images helped as an aide-memoire, and for the researcher were a useful way to maintain a focus on appearance during the interview. These were largely unstructured discussions but we would often suggest describing childhood memories of appearance as a useful starting point for people's narratives.

In these interviews we explored appearance more generally, so while we were interested in people's 'hair stories' we asked the person with dementia to lead the discussions and to let us know what was significant to them. We used these encounters as an opportunity to examine the subjective, social and cultural meanings that lay behind the way a person looked and were able to trace the emergence of certain appearance-practices over the life course and consider their significance in the present. For instance, Lily (pseudonym), a person with dementia who lives alone in sheltered accommodation, recalled a deprived working class upbringing where she was required to wear second-hand clothes throughout her childhood and the stigmatising responses to her appearance that she endured. Once at work and with an independent source of income, her appearance became an intensely meaningful vehicle for self-expression:

Lily: 'When I started work and I can remember the very first thing I had done. It was a perm and at the time they called it a bubble cut. When I think about it now it was horrendous'

Interviewer: 'And going to the hairdresser's regularly has that become a natural part of your life?'

Lily: 'Oh yes! Once I had money in my pocket I was every colour under the sun'

Interviewer: 'Really?'

Lily: 'They brought one out called Black Tulip and it was like a... it was very dark purple. But one thing I was very adventurous with was my hair. I'd have it permed, then I'd have it cut short, then I'd have it dyed. And I did all sorts with my hair. I wasn't very adventurous in any other way, but with my hair I did mess about, yeah'

The appearance biographies had a number of benefits to our understanding of appearance. Crucially, they provided opportunities for people with dementia to offer first-hand accounts of their appearance. The value of this is that it allowed us to go beyond our own readings and interpretations of a person's appearance to appreciate the sometimes highly personalised meanings it could hold, knowledge that would otherwise have been inaccessible to us as researchers. Sometimes vivid recollections of styles, colours, textures and scents revealed how sensory and embodied experiences are woven into life stories in meaningful ways. 
The interviews also had a relationship with the salon-based methods that underlined the value of mixing methods to better understand appearance. Hence, the appearance narratives provided a temporal and biographical context in which to think about the role of care-based hairdressing. They showed that a visit to the salon can offer an important source of continuity in people's lives, not only in terms of maintaining a certain look but continuity at an embodied and sensory level as well. The interviews situated people's bodies within a biographical and historical context. As a result we came to understand in more depth what people see when they look in the mirror - including glimpses of past selves, family resemblances and connections to other significant figures or events in their lives.

\section{Conclusions and implications for practice}

With the fieldwork completed, we have begun to reflect on the methods used for the Hair and Care project and in this concluding section wish to highlight certain insights relevant to dementia care practice.

In this paper we have argued for an understanding of appearance in dementia care as fluid and processual based upon the importance of attending to the different types work invested in its upkeep. This highlights the value of thinking critically about where appearance begins and ends in care and has led us to question the notion of appearance as fixed, and as an endpoint to care much in the way it is understood by the care providers observed in LeeTreweek's $(1994,1997)$ investigation. Instead, we have come to understand the 'doing of appearance' as integral to the performative nature of identities in care.

Research to date has underlined the ocularcentrism of dementia care, as it operates according to a system of visual signs and indicators in which the bodies of those being cared for serve a symbolic purpose (Lee-Treweek, 1997, Ward et al., 2008). Hence, for the Hair and Care project we have employed methods intended to help us understand how appearance feels. Our own participation in the salon process and in-situ approach to generating knowledge of it have revealed insights into 'the immediacy of the body' and its importance to both worker and client as a way of understanding the world (Kontos, 2006). While our efforts to find more embodied avenues to understanding the lives of people with dementia were at times experimental and exploratory, they point to important aspects of everyday life in care that are currently overlooked (see also Campbell, 2012). In particular, they have helped to understand how people use their senses to create meaningful worlds (Howes, 2003).

Our efforts to construct 'appearance biographies' in people with dementia involved attention to bodies in time. These interviews proved a useful method for gathering insights into people's embodied histories. A focus on appearance led to an appreciation of how people make sense of their changing bodies over time and the different influences upon their appearance that linked their bodies to wider structures of class, gender, sexuality and other forms of social difference. Importantly, in the course of recollecting sometimes vivid sensory memories and past experiences the interviews showed the multi-sensory nature of biographical narratives and the identities that emerge from them.

Our time in care-based salons also led to insights into the 'in-situ selves' of hairdressers and the people with dementia who visit them. Filming and observation of what people do as they are doing it showed the relationship to the social and material environment in which people are embedded and which shape their way of being in the world. As the filming captured the inter-corporeal engagement of worker and client we saw how appearance is 
jointly accomplished in the salon. Such insights underlined the significance of body work in care and its vital contribution to personhood and identity.

Above all, it is the relationship between the methods we have used that has set the scene for our analysis and understanding of appearance in dementia care. Different ways of seeing support different ways of understanding which in turn create possibilities for care practice. As Brownlie (2011) has argued, we should think reflexively about the different types of embodied knowledge and what they tells us; advice that applies to care practice as much as to research on dementia care. Using a mix of methods for the Hair and Care study we have underlined the multi-faceted nature of appearance in a way that resists any assumption about the relative significance of these different facets to people with dementia. Indeed, our time in the salon has underlined the diverse meanings that people attach to appearance and the work invested in maintaining it. On this basis we advocate the creative mixing of methods for dementia studies as an avenue to better understanding appearance in the lives of people with dementia and in this way contributing to the broader agenda of enhancing person-centred care.

\section{Funding}

This research has been funded by the Economic and Social Research Council (grant number: RES-06125-0484).

\section{Acknowledgement}

The authors wish to thank the editors for their useful comments on earlier drafts of this paper.

\section{References}

Bamford, C., \& Bruce, E. (2000). Defining the outcomes of community care: The perspectives of older people with dementia and their carers. Ageing and Society, 20, 543-570.

Biggs, S. (1997). Choosing not to be old? Masks, bodies and impression management in later life. Ageing and Society, 17(5), 533-553.

Bourdieu, P. (1977). Outline of a theory of practice. Cambridge, UK: Cambridge University Press.

Brownlie, J. (2011). 'Being there': multidimensionality, reflexivity and the study of emotional lives. The British Journal of Sociology, 62(3), 462-481.

Butler, J. (1993). Bodies that matter. Oxon, UK: Routledge.

Bytheway, B., \& Johnson, J. (1998). The sight of age. In S. Nettleton, \& J. Watson (Eds.), The body in everyday life. London, UK: Routledge.

Campbell, S. (2012). A close shave: masculinity and bodywork in dementia care. Graduate Journal of Social Science, 9(3). Retrieved from http://gjss.org/images/stories/volumes/9/3/Campbell.pdf.

Coupland, J. (2003). Ageist ideology and discourses of control in skincare product marketing. In J. Coupland, \& R. Gwyn (Eds.), Discourse, the body and identity. Basingstoke, UK: Palgrave Macmillan.

Faircloth, C. A. (Ed.) (2003). Aging bodies: Images and everyday experience. Walnut Creek, CA: AltaMira Press.

Featherstone, M., \& Hepworth, M. (1991). The mask of ageing and the postmodern lifecourse. In M. Featherstone, \& B. S. Turner (Eds.), The body: Social process and cultural theory. London, UK: Sage.

Furman, F. K. (1997). Facing the mirror: Older women and beauty shop culture. New York, NY: Routledge. 
Grosz, E. (1994). Volatile bodies. Bloomington, IN: Indiana University Press.

Howes, D. (2003). Sensual relations: Engaging the senses in culture and social theory. Ann Arbor: MI: University of Michigan Press.

Iltanen-Tahkavuori, S., Wikberg, M., \& Topo, P. (2012). Design and dementia: A case of garments designed to prevent undressing. Dementia, 11(1), 49-59.

Jackson, M. (1983). Knowledge of the body. Man, 18, 327-345.

Katz, S. (1996). Disciplining old age: The formation of gerontological knowledge. Charlottesville, VA; London, UK: University Press of Virginia.

Kontos, P. (2004). Ethnographic reflections on selfhood, embodiment and Alzheimer's disease. Ageing and Society, 24(6), 829-849.

Kontos, P. (2005). Multi-disciplinary configurations in gerontological research. In G. Andrews, \& D. Phillips (Eds.), Ageing and place: Perspectives, policy and practice. London, UK; New York, NY: Routledge: 24-35.

Kontos, P. (2006). Embodied selfhood: An ethnographic exploration of Alzheimer's disease. In A. Leibing, \& L. Cohen (Eds.), Thinking about dementia: Culture, loss and the anthropology of senility (pp. 195-217). New Brunswick, NJ: Rutgers University Press.

Lee-Treweek, G. (1994). Bedroom abuse: The hidden work in a nursing home. Generations Review, $4(2), 2-4$.

Lee-Treweek, G. (1997). Women, resistance and care: An ethnographic study of nursing auxiliary work. Work, Employment and Society, 11(1), 47-63.

Mason, J., \& Davies, K. (2011). Experimenting with qualitative methods: Researching family resemblance. In J. Mason, \& A. Dale (Eds.), Understanding social research: Thinking creatively about method (pp. 33-48). London, UK: Sage.

McColgan, G. (2005). A place to sit: Resistance strategies used to create privacy and home for people with dementia. Journal of Contemporary Ethnography, 34(4), 410-433.

Okely, J. (1978). Privileged, schooled and finished: Boarding education for girls. In S. Ardener (Ed.), Defining females: The nature of women in society (pp. 109-139). London, UK: Croom Helm.

Pink, S. (2005). Dirty laundry. Everyday practice, sensory engagement and the constitution of identity. Social Anthropology, 13(3), 275-290.

Shilling, C. (2003). The body and social theory. London, UK: Sage.

Topo, P., \& Iltanen-Tahkavuori, S. (2010). Scripting patienthood with patient clothing. Social Science and Medicine, 70(11), 1682-1689.

Turner, B. S. (1984). The body and society: Explorations in social theory. Oxford, UK: Blackwell.

Twigg, J. (2007). Clothing, age and the body: A critical review. Ageing and Society, 27(2), 285-305.

Twigg, J. (2010). Clothing and dementia: A neglected dimension? Journal of Aging Studies, 24, 223-230.

Ward, R., \& Campbell, S. (in press). An ethics journey: ethical governance of social research with vulnerable adults and the implications for practice. In M. Carey, \& L. Green (Eds.), Practical social work ethics: complex dilemmas in applied social care. Farnham, UK: Ashgate.

Ward, R., Vass, A. A., Aggarwal, N., Garfield, C., \& Cybyk, B. (2008). A different story: exploring patterns of communication in residential dementia care. Ageing and Society, 28(5), 629-651.

Wolkowtiz, C. (2006). Bodies at work. London, UK: Sage.

Ylanne, V. (Ed.) (2012). Representing ageing: Images and identities. Basingstoke, UK: Palgrave Macmillan.

\section{Author Biographies}

Richard Ward is a lecturer in dementia studies in the School of Applied Social Science at the University of Stirling. He is a qualified social worker with a particular interest in the care and 
support of people with dementia. Richard is currently leading an ESRC-funded study examining hairdressing and appearance-related support in dementia care. His other research interests include the role of neighbourhoods in the lives of people with dementia and their carers and the creative use of qualitative research methods in dementia studies. $\mathrm{He}$ is also a member of the British Sociological Society study group on Ageing, the Body and Society.

Sarah Campbell is a Research Associate at the University of Manchester. She has a background working with users of mental health services. She is the researcher for the Hair and Care project and studying for her $\mathrm{PhD}$ alongside this. Her doctoral studies are focused on considering masculinity and the experiences of men in dementia care. Sarah belongs to the Dementia and Ageing Research Team (DART) and has a particular interest in ethnographic methods in dementia studies. 